\title{
Trends in precipitation and temperature in Canberra
}

\author{
J. Hartigan $^{\text {a }}$, S. MacNamara ${ }^{\text {a }}$ and L. M. Leslie ${ }^{a}$ \\ ${ }^{a}$ School of Mathematical and Physical Sciences, University of Technology Sydney, Ultimo, New South \\ Wales 2007, Australia \\ Email: Joshua.Hartigan@student.uts.edu.au
}

\begin{abstract}
The current drought over much of southern and eastern Australia began not long after the Millennium Drought. While the Millennium Drought motivated Canberra to introduce measures to improve water availability, Canberra's population is increasing, placing greater strain on water resources. Further, its latitude is similar to other areas in the world in which drought frequency is increasing. Analysis of precipitation trends is required to assess how vulnerable an area might be to drought. In addition, if the mean temperature of a location is increasing, then the region might become more vulnerable to drought due to increased potential evaporation.
\end{abstract}

This statistical study utilises resampling methods to analyse trends in precipitation and mean temperature over Canberra. These resampling methods highlight the non-stationary nature of both precipitation and temperature time series. Minimal trends in precipitation were found, however there was an increasing trend in both mean maximum temperature $(\mathrm{p}$-value $=0.0028)$ and mean minimum temperature $(\mathrm{p}$-value $=0.0358)$ suggesting an increased vulnerability to drought for the region.

Numerous large-scale influences of climate such as the El-Niño Southern Oscillation (ENSO) have been used in the seasonal prediction of various atmospheric conditions. For example, an El-Niño event is typically associated with warmer and drier than average conditions over eastern Australia. Wavelet analysis provides a more thorough understanding of high- and low-frequency signals driving a non-stationary time series, and is applied here to identify potential drivers of the climate. Wavelet power spectra for precipitation reveal a statistically significant signal in the 2-7-yr range, which is typically indicative of influence by ENSO. Wavelet power spectra for mean maximum temperature reveal an increasing influence by ENSO, while mean minimum temperature reveals a decreasing influence. These findings exemplify how the influence of climate drivers can change over time. Accordingly, there is a need for recurrent analysis of the changing influence of climate drivers across Australia to improve skill in forecasts which utilise these climate drivers.

Keywords: Canberra, precipitation, temperature, drought, wavelet analysis, permutation testing 


\section{INTRODUCTION}

Drought has no universally accepted definition, though it can generally be considered as a period of deficient rainfall over some area, or some period in which water is in short supply (Bureau of Meteorology, 2012). Australia is no stranger to drought, with well-known examples of long-term drought periods including the Federation drought from 1895-1903, and the Millennium Drought from 1997-2009. The most recent drought has gained widespread media attention for its particularly sudden onset and extreme effects over much of southern and eastern Australia, occurring not long after the end of the Millennium Drought. On a global scale, regions of similar latitudes to southern Australia have also recently been affected by numerous droughts such as the Cape Town and California droughts (Richman and Leslie, 2015, 2018). Drought frequency has been observed to be increasing in these areas, so an understanding of the precipitation trends affecting southeast Australia is necessary to understand regions which might have enhanced vulnerability to drought.

Southeast Australia has observed a decrease in precipitation, particularly during autumn, due to increasing strength and poleward progression of the subtropical ridge (Timbal and Fawcett, 2013; Timbal and Drosdowsky, 2013; Post et al., 2014). However, decreasing precipitation is not the only factor which influences drought vulnerability. For example, the Millennium Drought was exacerbated by higher temperatures causing increased evaporation, which further reduced runoff (SEACI, 2010; Chiew et al., 2014). The majority of Australia has observed an increase in mean temperatures, with this increase projected to continue into the future (Reisinger et al., 2014). A combination of increasing temperatures and decreased precipitation can seriously tighten water availability, which can have significant effects including enhanced fire risk, reduced irrigation for agriculture, decreased sanitation and potentially serious effects on human health.

The Capital City of Australia, Canberra, is located inland from the east coast of Australia. Water availability in this region is entirely controlled by precipitation, which resulted in long-term water restrictions during the Millennium Drought. Following the drought, programs aimed at decreasing per capita water consumption were introduced, with significant reductions having occurred (Icon Water, 2019). However, this only provides a temporary solution to water availability as Canberra's population continues to grow (Australian Bureau of Statistics, 2018). Other projects include the enlargement of the Cotter dam, and development of a pipeline from the Murrumbidgee river to Googong dam (Icon Water, 2019). While the pipeline is an alternate source of water, it is still ultimately controlled by rain. The most recent drought led to only a short period of operation of the pipeline due to low water levels (Burgess, 2018), and as the Murrumbidgee river is a major contributor to the Murray-Darling basin, it might have limited utility during severe drought conditions. With no alternate sources of water than stored rainwater, understanding the trends in precipitation and temperature, as well as the climate drivers for this region, is necessary for proper water management. Advanced statistical techniques are employed in this study to understand these trends and potential climate drivers.

\section{DATA AND METHODOLOGY}

\subsection{Data}

Monthly mean maximum temperature (TMax), mean minimum temperature (TMin) and total monthly precipitation time series were obtained for Canberra $\left(35.3^{\circ} \mathrm{S}, 149.1^{\circ} \mathrm{E}\right)$ from the publically available Climate Data Online section of the Australian Bureau of Meteorology (BoM) website (http://www.bom.gov.au/ climate/data/index.shtml). Stations with relatively long records and few data gaps compared to other available stations in the area were chosen to represent Canberra. Any missing data points were filled using the moving average centred on the year for which the data was missing. Both precipitation and temperature time series start at 1938 as this is the earliest period in which both data were available for the region.

\subsection{Methodology}

This study examines precipitation and temperature trends over Canberra's wet season, and determines potential climate drivers of precipitation and temperature. The wet season was determined by comparing monthly averages of precipitation over the time series, which resulted in a wet season running from September through to February the following year. An overview of any trends present in the time series was first gained by graphing the time series with their percentiles. The data were then grouped into 20-year periods and bootstrap resampling was applied. This randomly selects 20 data points from within a specified 20 -year period, with replacement. This process was repeated 5000 times to estimate the underlying distribution, confirm non- 
stationarity of the data and gain a deeper understanding of any trends present within the data. Permutation testing was then applied with replacement to test if there is a statistically significant difference in the mean of the distribution underlying two 20 -year periods.

Wavelet analysis was performed on the time series following the approach of Torrence and Compo (1998). Wavelets are well-suited to the study of non-stationary time series as they provide an understanding of the time evolution of the periodicity of signals. This is useful for detecting potential climate drivers such as the El-Niño Southern Oscillation (ENSO) and analysing how the influence of climate drivers might change over time. Unlike Fourier and windowed Fourier transforms, the wavelet transform efficiently resolves both high- and low-frequency signals (Lau and Weng, 1995). This is done by stretching and translating local base functions in both space and time. This study used the Morlet wavelet which is described by

$$
\psi_{0}(\eta)=\pi^{-\frac{1}{4}} e^{i \omega_{0} \eta} e^{\frac{-\eta^{2}}{2}},
$$

where $\eta$ is a non-dimensional time parameter and $\omega_{0}$ is the non-dimensional frequency. The continuous wavelet for a time series, $x_{n}$, is given by

$$
W_{n}(s)=\sum_{n^{\prime}=0}^{N-1} x_{n^{\prime}} \psi^{*}\left(\frac{\left(n^{\prime}-n\right) \delta t}{s}\right),
$$

where $s$ is the wavelet scale, $n$ is a localised time index, $N$ is the number of data points in the time series and * is the complex conjugate. The wavelet transform is more efficiently computed in Fourier space rather than directly computing (2). The discrete Fourier transform of $x_{n}$ is

$$
\hat{x}_{k}=\frac{1}{N} \sum_{n=0}^{N-1} x_{n} e^{-2 \pi i k n / N},
$$

where $k=0,1, \ldots, N-1$ is the frequency index, and the Fourier transform of $\psi(t / s)$ is given by $\hat{\psi}(s \omega)$. By the convolution theorem, and taking the inverse Fourier transform, the wavelet transform is given by

$$
W_{n}(s)=\sum_{k=0}^{N-1} \hat{x}_{k} \hat{\psi}^{*}\left(s \omega_{k}\right) e^{-i \omega_{k} n \delta t},
$$

where

$$
\omega_{k}=\left\{\begin{array}{cll}
\frac{2 \pi k}{N \delta t} & : \quad k \leq \frac{N}{2} \\
\frac{-2 \pi k}{N \delta t} & : \quad k>\frac{N}{2} .
\end{array}\right.
$$

\section{Results}

\subsection{Evolution of precipitation}

Time series of precipitation and box plots of the bootstrapped mean wet season precipitation over 20-year periods for Canberra are provided in Figure 1. A table of p-values obtained by applying permutation tests to data and comparing the mean from 1978-1997 against 1998-2017 is provided in Table 1. Since the 1980s, the time series show a decrease in the number of years where recorded precipitation is above the 80th percentile. Apart from this, there is no clear trend in precipitation for Canberra with little overall change in the box plots. However, there is significant variation in each 20-year box plot compared to the previous 20-year period, which suggests some inter-decadal variability in precipitation and shows that the precipitation data is not stationary. Conceivably, hidden Markov models could be applied to simulate data sets such as these (e.g. Thyer and Kuczera, 2003). Other locations with a similar latitude such as Adelaide and Melbourne have begun exhibiting a decreasing trend in precipitation due to strengthening of the Hadley cell and the resultant poleward progression of cold fronts (Frederiksen et al., 2011; Timbal and Drosdowsky, 2013; Raut et al., 2014). This has been observed to occur during the autumn and winter months for these locations, which was not analysed in this study. It has been observed that, especially over spring and summer, pre-frontal troughs pass through Canberra and occasionally stall nearby. Canberra's proximity to the ocean allows considerable moisture buildup in the region, especially as sea surface temperatures continue to rise. Along with rising land temperatures, Canberra appears well-placed for increased instability and resulting convective storms and rain events. The pre-frontal troughs impacting Canberra might be the cause for no evident downward trend in recorded wet season precipitation. 

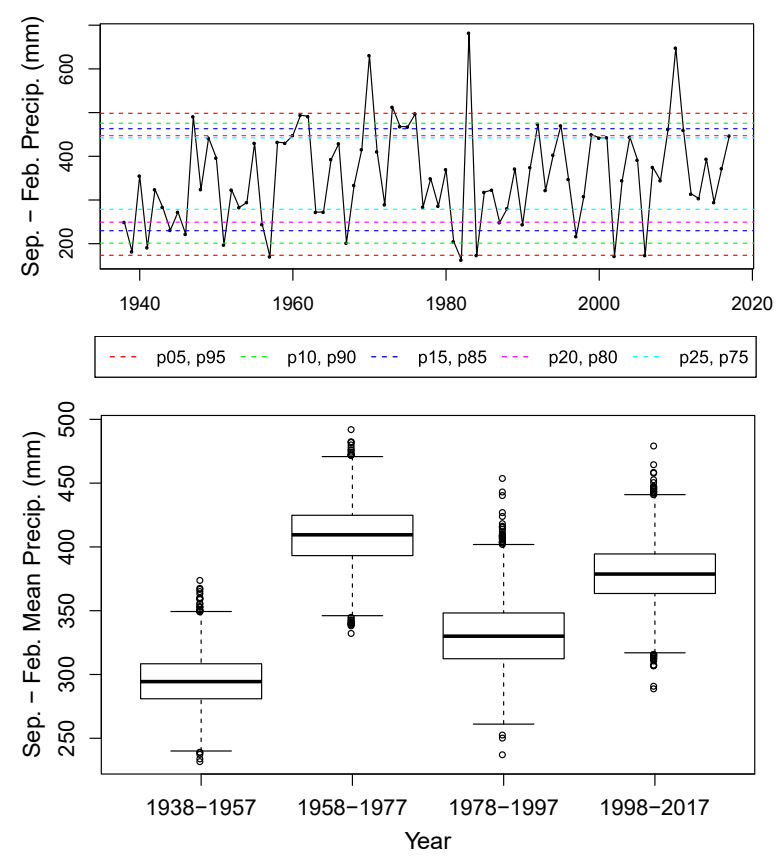

Figure 1. Time series of total wet season precipitation (top); and box plots of the bootstrapped mean wet season precipitation over 20-yr periods (bottom) for Canberra. Dashed lines indicate the 5th and 95th (bottom and top red), 10th and 90th (bottom and top green) 15th and 85th (bottom and top dark blue), 20th and 80th (bottom and top pink), and 25th and 75th percentiles (bottom and top light blue).

\subsection{Evolution of temperature}

Figure 2 is the same as Figure 1, but for the mean maximum temperature (TMax) and mean minimum temperature (TMin). If the trends in TMax and TMin are consistent, then the mean temperature should also roughly remain consistent with these trends.

TMax shows an increasing trend since the 1990s, with no years recorded below the 25 th percentile since 1992. As a result, there is a statistically significant difference between mean TMax over 19781997 and that in 1998-2017 (p-value $=0.0028$ ). TMin for Canberra has also increased, albeit this has been more gradual than TMax. The increase in TMin also started earlier than the increase in TMax. This is evident in the decreased frequency of years where TMin was recorded below the 10th percentile since the 1960s. Almost all years where TMin has been recorded above the 85th percentile were from the mid-1970s onwards, with an increased frequency in the number of years above the 85 th percentile from the late-1990s. There is a statistically significant increase in TMin between 1978-1997 and 1998-2017 (p-value $=0.0358$ ), highlighting the clear increasing trend in the 20-year box plots. As both TMax and TMin are increasing, it is suggested that the overall mean temperature in Canberra is increasing. The ACORN-SAT mean temperature data for Canberra was also analysed and confirms that mean temperature in Canberra is increasing (not shown), with a statistically significant difference in the mean temperature between 1978-1997 and 1998-2017 (p-value =0.0006).

\subsection{Wavelet analysis of precipitation}

Wavelet power spectra and global power spectra for Canberra's wet season precipitation are presented in Figure 3. The wavelet power spectra reveal a strong 2-7 yr signal. This is also evident in the global power spectra with two peaks over the 3-yr and 6-yr periods. No major change in influence of this signal is apparent over the time series. Power in the $2-7 \mathrm{yr}$ range is indicative of influence from the El Niño Southern Oscillation 

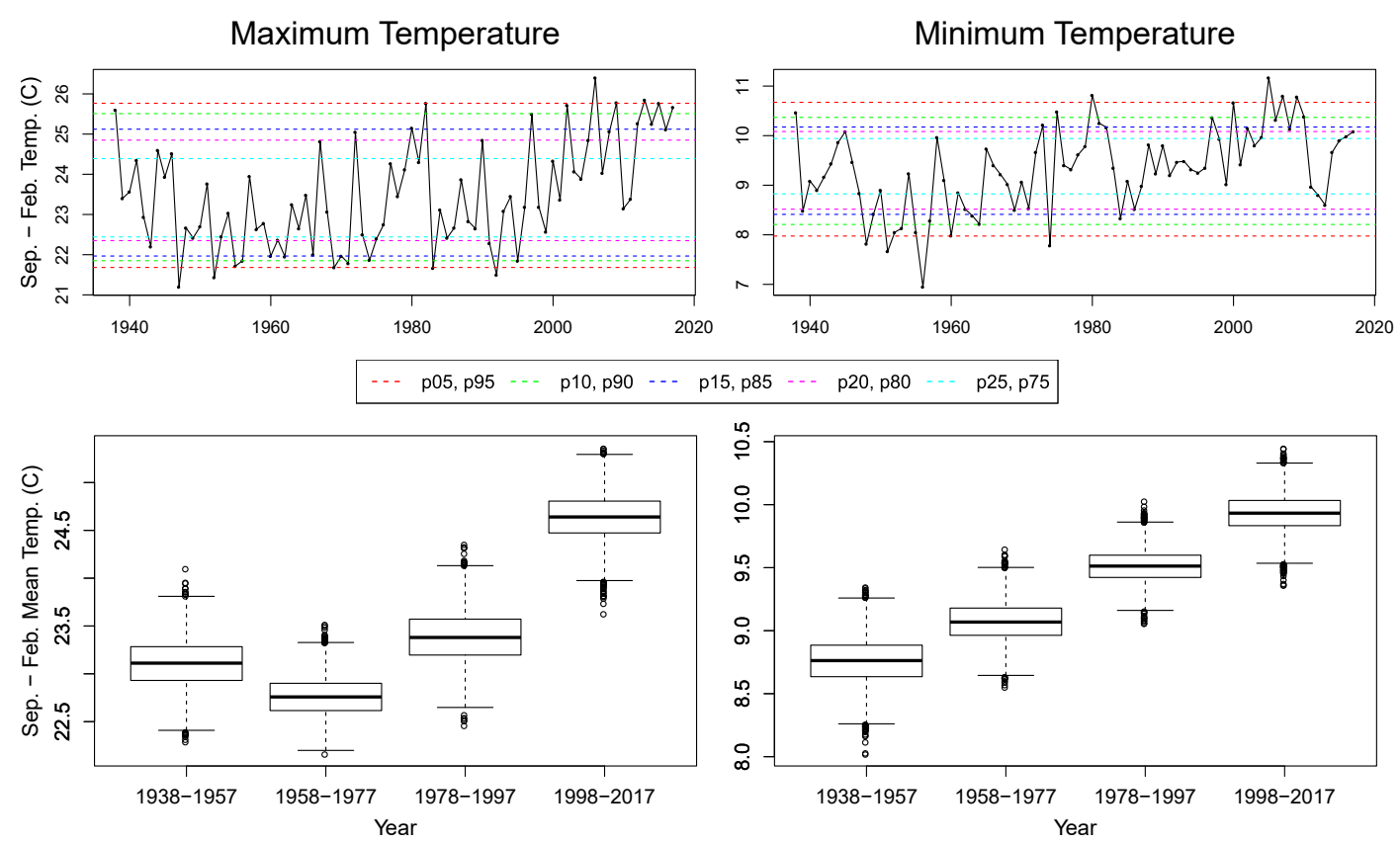

Figure 2. As in Figure 1 but for TMax (left) and TMin (right) during Canberra's wet season.

(ENSO). There is another peak around the 12-yr range in the global power spectra, and areas of high power around this range in the wavelet power spectrum. Like the box plots in Figure 1, this hints that there might be an inter-decadal influence on precipitation. These peaks are not statistically significant, however, due to the relatively short length of the time series.

\subsection{Wavelet analysis of temperature}

Wavelet power spectra also were computed for wet season TMax and TMin (Figure 4). The wavelet analysis and the box plots in Figure 2 are consistent with the mean, variance and the whole distribution varying in time with a variable climate, so the data can reasonably be described as non-stationary. There is high power for wet season TMax during the 2-4 yr period, which is most evident from the 1970s onwards. The reverse is true for TMin, with high power during the 2-4 yr period prior to the 1980 s. This changing power spectrum pattern roughly coincides with the global warming period. For TMin, the reduced power since the 1980s reveals that any natural variability is being masked. This $2-4 \mathrm{yr}$ period is indicative of an ENSO influence on Canberra's mean wet season temperatures, with more influence on TMax than TMin, especially since the 1980s. There is also some high power, though not statistically significant, around the 8-10 yr period, mainly for TMin but also

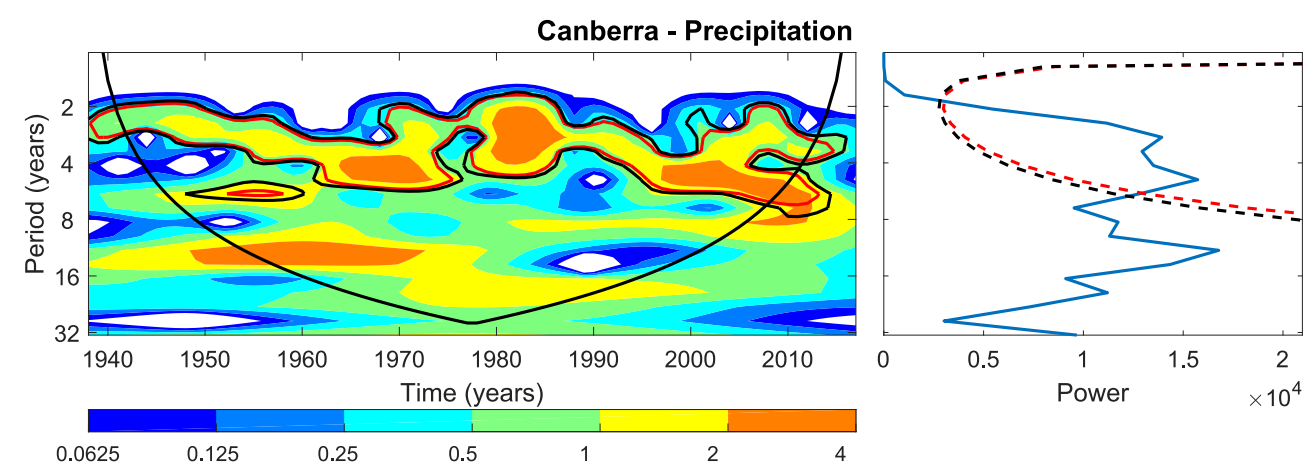

Figure 3. Wavelet analysis for total wet season precipitation for Canberra. Low values (blue) in the wavelet power spectrum (left) indicate low variability while high values (red) indicate high variability. Peaks in the global power spectrum (right) on the right panel indicate high variability. The dashed/solid red line indicates the $95 \%$ confidence level, while the dashed/solid black line indicates the $90 \%$ confidence level in each plot. 

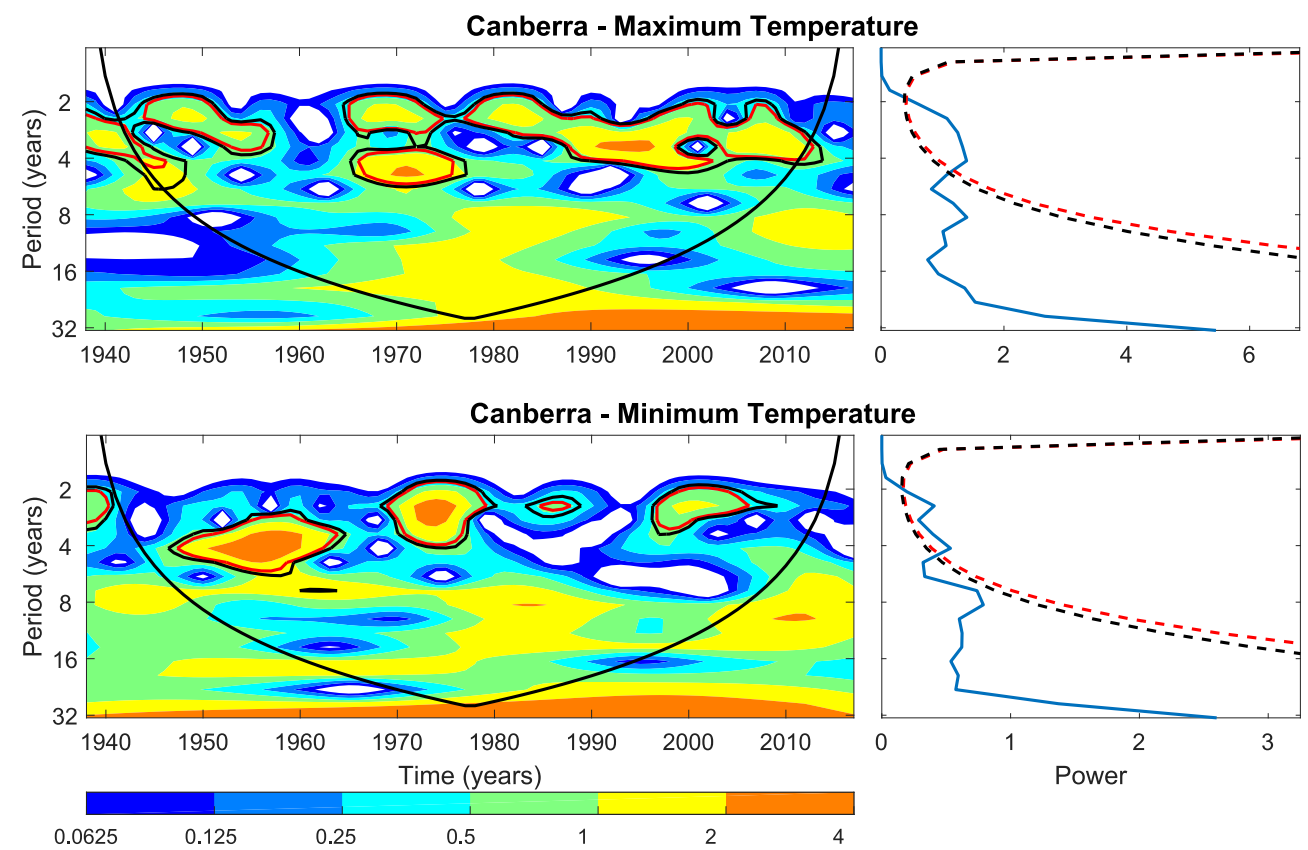

Figure 4. As in Figure 3, but for TMax (top row) and TMin (bottom row).

TMax, since the 1980s. Power spectra for both temperature time series appear to be less influenced by climate drivers than the precipitation time series.

\section{Discussion And Conclusions}

Over the past two years, much of southern and eastern Australia has experienced rainfall that is very much below average. This has resulted in a sharp and widespread drought, following soon after the Millennium Drought which affected southern Australia from 1997-2009. In Canberra, numerous initiatives were developed in response to the Millennium Drought, aimed at decreasing per capita water consumption and increasing the catchment of precipitation. In this study, precipitation and temperature time series were analysed using statistical resampling methods. These time series were found to be non-stationary, so wavelets were used to identify potential climate drivers of both precipitation and temperature.

Precipitation has been observed to decrease across southeast Australia, particularly during autumn (Timbal and Fawcett, 2013). While there has been a decrease in the number of wet seasons with precipitation recorded above the 80th percentile, there appears to be no significant increasing or decreasing trend in precipitation over Canberra's wet season, defined here as the months from September through to February. It is hypothesised that pre-frontal troughs, and Canberra's location close to the Tasman Sea provide an ideal synoptic situation conducive to precipitation events. Detailed analysis is required to better understand the synoptic-scale conditions associated with Canberra's precipitation during the wet season. Meanwhile, both mean maximum and minimum temperature during Canberra's wet season have been found to increase. This suggests that the mean temperature in Canberra is also increasing, similar to what has been observed across Australia (Reisinger et al., 2014). A decrease in the frequency of high rainfall wet seasons, combined with increasing mean temperatures could cause significant decreases in runoff (Chiew et al., 2014), which would have serious implications for Canberra's water security.

ENSO has previously been associated with both temperature and precipitation variability along eastern Australia, with El-Niño events causing increased risk of drought and La-Niña events increasing the risk of floods (McBride and Nicholls, 1983; Ropelewski and Halpert, 1987; Halpert and Ropelewski, 1992). Wavelet analysis revealed that precipitation in Canberra is consistently reliant on ENSO, which has a period of 2-7 years. Mean maximum temperature appears to be increasingly controlled by ENSO since the 1970s, while mean minimum temperature has become less controlled. This change in the influence of ENSO reflects the non-stationary nature of the time series. Despite ENSO being considered a good predictor for various weather 
phenomena, there are a plethora of other climate drivers that play a role in the atmospheric conditions at a location. Further research is necessary to understand the relative role of each climate driver. This study highlights the importance of understanding how the influence of climate drivers changes over time. Without knowledge of how each climate driver influences certain atmospheric conditions, forecasts which utilise these climate drivers can become considerably less skillful, which can have far-reaching economic and societal impacts.

\section{ACKNOWLEDGEMENTS}

This research is supported by an Australian Government Research Training Program Scholarship. Shev MacNamara acknowledges funding from the Australian Research Council Centre of Excellence for Mathematical and Statistical Frontiers. We thank an anonymous reviewer for their suggestions which have improved the quality of the manuscript.

\section{REFERENCES}

Australian Bureau of Statistics (2018). Australian Demographic Statistics. Technical report. 60 pp. Bureau of Meteorology (2012). Climate Glossary - Drought.

Burgess, K. (2018). Canberra's emergency water pipeline makes short-lived debut. The Canberra Times.

Chiew, F., N. Potter, J. Vaze, C. Petheram, L. Zhang, J. Teng, and D. Post (2014). Observed hydrologic non-stationarity in far south-eastern Australia: implications for modelling and prediction. Stochastic Environmental Research and Risk Assessment 28, 3-15.

Frederiksen, J., C. Frederiksen, S. Osbrough, and J. Sisson (2011). Changes in Southern Hemisphere rainfall, circulation and weather systems. In 19th International Congress on Modelling and Simulation, pp. 27122718.

Halpert, M. and C. Ropelewski (1992). Surface Temperature Patterns Associated with the Southern Oscillation. Journal of Climate 5, 577-593.

Icon Water (2019). Water for Generations.

Lau, K. and H. Weng (1995). Climate Signal Detection Using Wavelet Transform: How to Make a Time Series Sing. Bulletin of the American Meteorological Society 76, 2391-2402.

McBride, J. and N. Nicholls (1983). Seasonal Relationships between Australian Rainfall and the Southern Oscillation. Monthly Weather Review 111, 1998-2004.

Post, D., B. Timbal, F. Chiew, H. Hendon, H. Nguyen, and R. Moran (2014). Decrease in southeastern Australian water availability linked to ongoing Hadley cell expansion. Earth's Future 2, 231-238.

Raut, B., C. Jakob, and M. Reeder (2014). Rainfall Changes over Southwestern Australia and Their Relationship to the Southern Annular Mode and ENSO. Journal of Climate 27, 5801-5814.

Reisinger, A., R. Kitching, F. Chiew, L. Hughes, P. Newton, S. Schuster, A. Tait, and P. Whetton (2014). Climate Change 2014: Impacts, Adaptation, and Vulnerability. Part B: Regional Aspects. Contribution of Working Group II to the Fifth Assessment of the Intergovernmental Panel on Climate Change. Technical report. 1371-1438 pp.

Richman, M. and L. Leslie (2015). Uniqueness and Causes of the California Drought. Procedia Computer Science 61, 428-435.

Richman, M. and L. Leslie (2018). The 2015-2017 Cape Town Drought: Attribution and Prediction Using Machine Learning. Procedia Computer Science 140, 248-257.

Ropelewski, C. and M. Halpert (1987). Global and Regional Scale Precipitation Associated with the El Niño/Southern Oscillation. Monthly Weather Review 115, 1606-1626.

SEACI (2010). Climate Variability and Change in South-eastern Australia: A Synthesis of Findings from Phase 1 of the South Eastern Australian Climate Initiative (SEACI). CSIRO.

Thyer, M. and G. Kuczera (2003). A hidden Markov model for modelling long-term persistence in multi-site rainfall time series 1. Model calibration using a Bayesian approach. Journal of Hydrology 275, 12-26.

Timbal, B. and W. Drosdowsky (2013). The relationship between the decline of Southeastern Australian rainfall and the strengthening of the subtropical ridge. International Journal of Climatology 33, 1021-1034.

Timbal, B. and R. Fawcett (2013). A Historical Perspective on Southeastern Australian Rainfall since 1865 Using the Instrumental Record. Journal of Climate 26, 1112-1129.

Torrence, C. and G. Compo (1998). A Practical Guide to Wavelet Analysis. Bulletin of the American Meteorological Society 79, 61-78. 